\title{
Confirmation of mosaicism and uniparental disomy in amniocytes, after detection of mosaic chromosome abnormalities in chorionic villi
}

\author{
Francesca R Grati ${ }^{*}{ }^{1}$, Beatrice Grimi ${ }^{1}$, Giuditia Frascoli ${ }^{1}$, Anna Maria Di Meco ${ }^{1}$, \\ Rosaria Liuti ${ }^{1}$, Silvia Milani ${ }^{1}$, Anna Trotta ${ }^{1}$, Francesca Dulcetti ${ }^{1}$, Enrico Grosso ${ }^{2}$, \\ Monica Miozzo ${ }^{3}$, Federico Maggi ${ }^{1}$ and Giuseppe Simoni ${ }^{1,3}$
}

\footnotetext{
${ }^{1}$ Units of Cytogenetics and Molecular Biology, TOMA Laboratory, Busto Arsizio, Varese, Italy; ${ }^{2}$ SCDU Genetica Medica, Azienda Ospedaliera San Giovanni Battista, Turin, Italy; ${ }^{3}$ Cattedra di Genetica Medica, Dipartimento di Medicina, Chirurgia e Odontoiatria, University of Milan, Milan, Italy
}

Chromosome mosaicism is detected in about $1-2 \%$ of chorionic villi samples (CVS), and may be due to a postzygotic nondisjunction event generating a trisomic cell line in an initially normal conceptus (mitotic origin) or the postzygotic loss of one chromosome in an initially trisomic conceptus (meiotic origin and trisomy rescue). Depending on the distribution of the abnormal cell line, the mosaic can be confined to the placenta (CPM) or generalised to the fetus (TFM, true fetal mosaicism). Trisomy rescue could theoretically be associated with a $33.3 \%$ probability of uniparental disomy (UPD) in the fetus. The aim of this study was to determine the risk of fetal involvement in a cohort of numerical and structural chromosome mosaics revealed in chorionic villi by means of combined direct and long-term culture analyses; we also determined the incidence of UPD associated with mosaic aneuploidies and supernumerary markers involving imprinted chromosomes. A total of 273 of a consecutive series of 15109 CVS evaluated during a period of 5 years showed a mosaic condition in direct preparations and/or long-term cultures; confirmatory amniocentesis was performed in 203 cases. The abnormal cell line was extended to the fetus in $12.8 \%$ cases in terms of structural and numerical abnormalities involving autosomes and sex chromosomes; the risk of TFM varied and depended on the placental tissue distribution of the abnormal cell line. One of the 51 cases in which the mosaic involved an imprinted chromosome showed UPD, thus indicating a risk of $1.96 \%$.

European Journal of Human Genetics (2006) 14, 282-288. doi:10.1038/sj.ejhg.5201564; published online 18 January 2006

Keywords: confined placental mosaicism; true fetal mosaicism; uniparental disomy; aneuploidies; chromosome rearrangements

\section{Introduction}

Chromosome mosaicism is defined as the presence of two or more karyotypically different cell lines in the fetoplacental unit arising from a single zygote, and is detected

*Correspondence: Dr FR Grati, TOMA, Advanced Biomedical Assays, S.p.A., Via Ferrer 25/27, 21052 Busto Arsizio VA, Italy.

Tel: + 390331 652911; Fax: + 390331 652919;

E-mail: fgrati@tomalab.com

Received 24 June 2005; revised 16 November 2005; accepted 24 November 2005; published online 18 January 2006 in about $1-2 \%$ of chorionic villous samples (CVS) used to assess the fetal karyotype. ${ }^{1-9}$

It develops as a result of a viable somatic postzygotic mutational event as: when the error occurs soon after fertilisation (before the differentiation of the trophoblast and the inner cell mass), the mosaic can be generalised to both placental and fetal tissues; when it occurs at a later gestational stage (after the separation of the fetal and placental compartments), the abnormal cells may be confined to the placenta (confined placental mosaicism, CPM) or fetus, but not necessarily both. ${ }^{10}$ 
There are three types of CPM, each of which affects a different placental cell lineage. ${ }^{11,12}$ In type I, the abnormal cell line is confined to the cytotrophoblast; in type II, it only affects the stromal villous core (mesenchyme); and in type III, it involves both tissues. The karyotype of the two placental cell lineages can be obtained by means of direct preparations or short-term cultures (cytotrophoblast), or long-term incubations (mesenchyme) of the chorionic villi.

Disomy-trisomy mosaicism may be due to one of these two different mechanisms: a mitotic postzygotic nondisjunction event generating a trisomic cell line in an initially normal conceptus (mitotic origin); or a postzygotic loss of the supernumerary chromosome, which restores disomy in a trisomic conceptus (meiotic origin and trisomy rescue). Type I and II CPMs are mainly mitotic in origin, and type III is mainly meiotic. This last condition is associated with an increased risk of pregnancy complications and a theoretical probability of $33.3 \%$ of uniparental disomy (UPD) in the 'rescued' diploid fetus, ${ }^{13}$ because of the presence of one chromosome pair derived from only one parent. ${ }^{14}$ In the case of a mitotic origin, the risk of UPD is very low. ${ }^{15}$ The phenotype effects of UPDs depend on the presence of imprinted genes in the involved chromosome pair and the reduction of autosomal recessive mutations to homozygosity. ${ }^{16-20}$ UPDs and their phenotype effects have been described for a number of chromosomes. $^{21}$

The aim of this study was to determine the risk of true fetal mosaicism (TFM) in a large cohort of numerical and structural chorionic villous mosaics taken from a large series of CVS systematically analysed by means of direct chromosome preparations and long-term cultures. We also tried to determine the incidence of UPDs associated with mosaic aneuploidies and supernumerary marker chromosomes.

\section{Materials and methods \\ Samples and procedures}

Over a period of 5 years, we analysed a total of 15109 CVS received from 85 public and private centres in the north of Italy. All the cases underwent similar procedures using homogeneous evaluation criteria: a total of at least 30 metaphases for each CVS was analysed by means of direct (a minimum of 15 cells) and long-term in situ culture preparations (a minimum of six cells).

A condition of CV mosaicism was defined as the presence of at least two cells showing the same chromosomal alteration (trisomy or a structural rearrangement). In the case of monosomy, at least three cells with the same abnormality were required (ISCN 1995). The samples with a single abnormal metaphase were not included in the study. In all the cases of CV mosaicisms, a confirmatory amniocentesis was recommended for further cytogenetic investigations, and UPD testing when the cytogenetic abnormality (trisomy or a supernumerary marker) involved imprinted chromosomes. Karyotyping from amniotic fluid (AF) was performed by analysing at least 40 metaphases from a minimum of 20 colonies taken from more than one culture.

TFM was defined as the presence of at least two metaphases showing the same abnormality as that previously observed in $\mathrm{CV}$, and a nonmosaic feto-placental discrepancy as the homogeneous presence of the abnormal cell line in one of the analysed tissues.

\section{DNA analysis}

UPD testing was systematically carried out on genomic DNAs from parental peripheral blood lymphocytes (PBLs) and amniocytes whenever a feto-placental discrepancy was found and the abnormal cell line involved a trisomy for chromosomes $2,6,7,11,14,15,16$ or 20 , or had a supernumerary marker chromosome derived from chromosome 14 or 15 (invdup 14 or 15). The DNAs were extracted using the QIAamp DNA Mini Kit (Qiagen Inc., Chatsworth, CA, USA). UPD was investigated by means of parent-tofetus segregation analysis using a panel of four to five highly polymorphic short tandem repeat (STR) markers located along the involved chromosome obtained from the Genome Database (GDB, http://www.gdb.org/) and the NCBI (http://www.ncbi.nlm.nih.gov/genomes/static/euk_g. html), and confirmed when at least two informative markers indicated the presence of uniparental inheritance, and at least five STRs mapping to other chromosomes confirmed the paternity. The UPD cases were further investigated by means of additional STR markers distributed along the chromosome in order to distinguish heteroand isodisomy patterns. The PCR products were separated on ethidium bromide-stained denaturing $10 \%$ polyacrylamide gel as described by Grati et al (2000), ${ }^{22}$ or run on a fluorescent capillary system (ABI PRISM 310, ABI, Foster City, CA, USA) after fluorescent PCR using one ABI-dye labelled primer.

\section{Results}

CV mosaicism was found in 273 (1.81\%) of 15109 CVS analysed between 2000 and 2005. The karyotype of 203 cases was subsequently investigated on amniocytes and classified into six different classes of mosaicism on the basis of tissue involvement. In order to facilitate comparison, our data are given in the same format as that used in the EUCROMIC study. ${ }^{23}$

As can be seen in Table 1, the mosaicism was confined to the placenta in 177 cases (87.2\%): 39.9\% type I, 40.4\% type II and $6.9 \%$ type III; in the remaining $12.8 \%$ of cases, the presence of the chromosome abnormality was also confirmed in amniocytes $\left(\mathrm{TFM}^{23}\right)$. None of our confirmatory AF analyses detected a single cell carrying the same abnormality as that found in CV. 
Table 1 Incidences of the different types of mosaicisms (CPM and TFM) found after chrionic villous and amniocytes karyotyping

\begin{tabular}{lllllr}
\hline Type & Nature & Trophoblast (direct) & Mesenchyme (culture) & Amniocytes & Relative frequencies (\%) \\
\hline I & CPM & Abnormal & Normal & Normal & $39.9(81 / 203)$ \\
II & CPM & Normal & Abnormal & Normal & $40.4(82 / 203)$ \\
III & CPM & Abnormal & Abnormal & Normal & $6.9(14 / 203)$ \\
IV & TFM & Abnormal & Normal & Abnormal & $1(2 / 203)$ \\
V & TFM & Normal & Abnormal & Abnormal & $5.9(12 / 203)$ \\
VI & TFM & Abnormal & Abnormal & Abnormal & $5.9(12 / 203)$ \\
\hline
\end{tabular}

Table 2 Probabilities of confirmation on amniocytes of Mosaic or Non Mosaic abnormal cell line considering the different combinations of the affected placental tissues

\begin{tabular}{|c|c|c|}
\hline $\begin{array}{l}\text { Trophoblast } \\
\text { (direct) }\end{array}$ & $\begin{array}{l}\text { Mesenchyme } \\
\text { (culture) }\end{array}$ & Confirmation \\
\hline A & $\mathbf{N}$ & $2.4 \%(2 / 83)$ \\
\hline $\mathrm{MA}$ & $\mathrm{N}$ & $\begin{array}{l}\text { Type IV } / \text { Type I+IV = } 1 / \\
64+1=1.5 \%\end{array}$ \\
\hline NMA & $\mathrm{N}$ & $1 / 16+1=5.9 \%$ \\
\hline & A & $12.8 \%(12 / 94)$ \\
\hline $\mathrm{N}$ & MA & $\begin{array}{l}\text { Type V/Type II+V 4/ } \\
71+4=5.3 \%\end{array}$ \\
\hline$N$ & NMA & $8 / 11+8=42.1 \%$ \\
\hline A & & $46.1 \%(12 / 26)$ \\
\hline MA & MA & $\begin{array}{l}\text { Type VI/Type III+VI=7/ } \\
5+7=58.3 \%\end{array}$ \\
\hline NMA & MA & $1 / 8+1=11.1 \%$ \\
\hline MA & NMA & $4 / 0+4=100 \%$ \\
\hline NMA & NMA & $0 / 1+0=0 \%$ \\
\hline
\end{tabular}

$\mathrm{A}=$ Abnormal; $\mathrm{N}=$ Normal; $\mathrm{MA}=$ Mosaic Abnormality; $\mathrm{NMA}=$ Non Mosaic Abnormality.

The chances of fetal involvement assessed on the basis of the CV mosaicism (Table 2) were $46.1 \%$ in the case of type III, but much less in the case of type II (12.8\%) and type I $(2.4 \%)$. The separation of mosaic (MA) and nonmosaic abnormalities (NMA) showed that the risk of fetal confirmation was $42.1 \%$ in the case of an NMA in the mesenchyme alone, and $58.3 \%$ in case of an MA in both placental tissues; this latter probability was $100 \%$ when the mesenchyme was homogeneously involved (Table 2).

\section{True fetal mosaicism of aneuploidies}

Analysis of the distribution of abnormalities and the chromosomes involved (Table 3 ) showed that aneuploidies were more frequent than structural rearrangements $(79.3 \%$ vs $20.7 \%$ ) and that the most frequent numerical abnormalities involved sex chromosomes (36 cases), tetraploidies (14), and chromosomes 7 (17), 21 (12), 2 (11), 18 (11) and 13 (9). The aneuploidies confirmed in the fetus involved chromosomes X/Y (14 cases), 18 (4) and 21 (4). Trisomy 18 was only found in the case of an NMA.

\section{True fetal mosaicism of structural abnormalities}

In the presence of structural rearrangements, we investigated whether they involved an additional marker chro- mosome $(47,+$ mar) or not $(46$, rearr): $3 / 11$ cases in the former group (27.3\%) were confirmed in $\mathrm{AF}$, and $1 / 31$ cases in the latter group (3.2\%) showed an NMA in amniotic cells as previously found in the mesenchyme. The details concerning the type of structural rearrangement, the number of cells examined, the tissue involved, and the indication for prenatal diagnosis are shown in Table 4.

\section{Confined placental mosaicism and UPD screening}

Among the CPM cases, the 46, rearr structural rearrangements and chromosome 2, 7 and 21 trisomies were preferentially confined to the mesenchyme, whereas those of chromosome 3 and the $\mathrm{X}$ chromosome aneuploidies were preferentially confined to the cytotrophoblast (Table 3). The abnormal cell line was more often homogeneously present in the cytotrophoblast ( 24 cases) than in the mesenchyme (11 cases) (Table 3 ). One case of NMA trisomy 22 involving both placental cell types was associated with a normal male karyotype in amniocytes; ultrasound (US) evaluation only revealed the presence of intra-uterine growth retardation (IUGR).

UPD testing was performed in 47 cases of trisomy involving imprinted chromosomes and four cases with satellited supernumerary markers derived from chromosomes 15 or 14 . UPD was found in only one instance (1.96\%); a type I CPM with a trisomy 14 cell line in a pregnant 43-year-old woman. In the 12 th week of gestation (wg), CV chromosome analysis was performed on a total of 36 metaphases; 28 obtained directly and eight after culture. Direct chromosome analysis showed a 47,XY, + 14 karyotype in 12 of the 28 metaphases; the cells obtained after culture of the mesenchymal stroma showed a normal karyotype in all of the metaphases. After counselling, amniocentesis was performed in the $15 \mathrm{th}+4 \mathrm{wg}$, and a $46, \mathrm{XY}$ karyotype was found in all of the 40 metaphases scored from 23 colonies from five cultures. The US revealed a normal amount of amniotic fluid, a biparietal diameter of $31.6 \mathrm{~mm}$ (50th percentile), an abdominal circumference of $9 \mathrm{~cm}$ (25th percentile), a cranial circumference of $12.3 \mathrm{~cm}$ (50th percentile), a femoral length of $17.1 \mathrm{~mm}(<5 \mathrm{th}$ percentile) and a humeral length of $17.2 \mathrm{~mm}$ (50th percentile). The decrease in femoral length was 2 SD. Eight of the 15 STR markers analysed were informative in defining matUPD. All of the three STRs in the pericentromeric 
Table 3 Distribution of specific chromosomal alterations in the different types of mosaicisms (CPM and TFM)

\begin{tabular}{|c|c|c|c|c|c|c|c|}
\hline \multirow[b]{2}{*}{ Chr abnormality } & \multicolumn{3}{|c|}{ CPM number of cases } & \multicolumn{3}{|c|}{ TFM number of cases } & \multirow[b]{2}{*}{ Total (203) } \\
\hline & Type I & Type II & Type III & Type IV & Type V & Type VI & \\
\hline+1 & & 1 & & & & & 1 \\
\hline+2 & 1 & $8+2^{\wedge}$ & & & & & 11 \\
\hline+3 & 6 & & 1 & & & & 7 \\
\hline+4 & 1 & & & & & & 1 \\
\hline+6 & & 1 & $1^{C}$ & & & & 2 \\
\hline+7 & $5+1^{\wedge}$ & $9+1^{\wedge}$ & 1 & & & & 17 \\
\hline+8 & $1+2^{\wedge}$ & 1 & 1 & & & & 5 \\
\hline+9 & 2 & 3 & & & & & 5 \\
\hline+10 & 1 & 2 & & & & & 3 \\
\hline+11 & 2 & & & & & & 2 \\
\hline+12 & 1 & & & & & & 1 \\
\hline+13 & 3 & 3 & $1+2^{C}$ & & & & 9 \\
\hline+14 & $1+1^{*}$ & & & & & & 2 \\
\hline+15 & $2+1^{\wedge}$ & 1 & $1^{\mathrm{C}}$ & & & & 5 \\
\hline+16 & $1+1^{\wedge}$ & 1 & & & & & 3 \\
\hline+17 & & 1 & & & & & 1 \\
\hline+18 & 4 & 3 & & & $\left(3^{\wedge}\right)$ & $\left(1^{\mathrm{M}}\right)$ & 11 \\
\hline+20 & 1 & 2 & $2^{C}$ & & & & 5 \\
\hline+21 & 1 & $6+1^{\wedge}$ & & & $1+\left(2^{\wedge}\right)$ & $\left(1^{c}\right)$ & 12 \\
\hline+22 & & & $1^{\mathrm{CM}}$ & & & & 1 \\
\hline-22 & & 1 & & & & & 1 \\
\hline$+X$ & $3 X Y+1 X X+1^{\wedge} X X$ & $1 X Y$ & & & $1 \mathrm{XY}$ & $1 X X+\left(1^{M} X Y\right)$ & 9 \\
\hline$+Y$ & & & & & & $1 X Y$ & 1 \\
\hline$-X$ & $6+2^{\wedge}$ & 2 & & & $2+1^{\wedge}$ & 1 & 14 \\
\hline$-Y$ & 2 & 3 & 1 & & $1^{\wedge}$ & $1+\left(1^{\mathrm{M}}\right)$ & 9 \\
\hline$X / X X / X X X$ & & & & & & $2+1^{\mathrm{M}^{\prime}}$ & 3 \\
\hline 47,+mar & $4+1^{\wedge}$ & $2+1^{\wedge}$ & & $1^{\wedge}+1$ & & 1 & 11 \\
\hline 46 , rearr & $7+5^{\wedge}$ & $14+4^{\wedge}$ & & & $\left(1^{\wedge}\right)$ & & 31 \\
\hline Tetraploidy & $4+4^{\wedge}$ & $2+2^{\wedge}$ & $2^{\mathrm{C}}$ & & & & 14 \\
\hline Multiple trisomy & 2 & 4 & & & & & 6 \\
\hline
\end{tabular}

* = UPD case.

( ) = nonmosaic abnormal cell line in the fetus.

$\wedge=$ nonmosaic abnormal cell line in placenta (in cytotrophoblast $\left(^{C}\right)$ or in mesenchyme $\left({ }^{M}\right)$ ).

$\mathrm{XY} / \mathrm{XX}=$ normal karyotype associated with the $+\mathrm{X} /+\mathrm{Y}$ cell line.

region were isodisomic, and one telomeric marker (D14S68) showed heterodisomy (Figure 1). After counselling, the parents refused any further clinical and US evaluations; the pregnancy was voluntarily interrupted in the $16 \mathrm{th}+5 \mathrm{wg}$ and the parents did not consent to an autopsy.

\section{Discussion}

This study is based on 273 cases of mosaicism found in a consecutive series of 15109 CVS analyses of direct preparations and/or long-term CV cultures made in our laboratory over a period of 5 years. Confirmatory amniocentesis was suggested in all instances, and the amniotic fluid samples of 203 cases were also analysed in our laboratory; in the remaining 70 cases, a therapeutic abortion was performed without further investigation, or the confirmatory AF samples were analysed elsewhere.

\section{True fetal mosaicism: risk assessment}

In line with the results of previous studies, the frequency of $\mathrm{CV}$ mosaicism was $1.81 \%$, and the risk of fetal involvement depended on the distribution of the abnormal cell line in placental tissue; ${ }^{1-9}$ for example, in type III CV mosaicism, the risk of TFM was $58.3 \%$ but increased to $100 \%$ when the abnormal cell line was homogeneously present in the mesenchyme (Table 2).

Our TFM percentages are different from those based on the EUCROMIC data reported by Hahnemann and Vejerslev, ${ }^{23}$ who did not find any case of TFM associated with type I CV mosaicism, whereas we found two cases: one with an NMA 47,+ mar and the other with an MA $47,+$ mar, on the basis of which, we calculated a percentage risk of $2.4 \%$ (Table 2 ). In addition, our data indicate that the percentage of TFM in cases of type II CV NMA is lower than that previously reported $(42.1 \%$ vs $83.3 \%)$. These differences can be explained by the fact that ours was a single-centre analysis and all of the samples underwent the same procedures using the same evaluation criteria.

Aneuploidies TFMs were found in the case of aneuploidies compatible with a viable fetus; trisomy 18 and 21 and $\mathrm{X}-\mathrm{Y}$ aneuploidies. ${ }^{23}$ Unlike other authors, ${ }^{23,24}$ we did not recover any autosomal trisomies other than 21,18 and 13 
Table 4 Description of the mosaic cases involving structural rearrangements. Karyotypes include in square brackets the number of the examined cells

\begin{tabular}{|c|c|c|c|c|}
\hline \multirow[b]{2}{*}{ Indication } & \multirow[b]{2}{*}{ Mosaic Karyotype } & \multicolumn{2}{|c|}{ CVS } & \multirow[b]{2}{*}{ Amniocytes confirmation } \\
\hline & & Cytotrophoblast & Mesenchyme & \\
\hline PPP $(45, \times 0)$ & $46, X X / 47, X X,+\operatorname{mar}(B S)$ & $N[0 / 19]$ & MA [3/8] & \\
\hline AMA & $46, \mathrm{XY} / 47, \mathrm{XY},+\operatorname{mar}(\mathrm{BS})$ & NMA [18/18] & $\mathrm{N}[0 / 13]$ & $46, \mathrm{XY} / 47, \mathrm{XY},+\mathrm{mar}$ \\
\hline AMA & $46, \mathrm{XX} / 47, \mathrm{XX},+\operatorname{mar}(\mathrm{NS})$ & $\mathrm{N}[0 / 22]$ & MÁ [2/9] & \\
\hline AMA & $46, \mathrm{XX} / 47, \mathrm{XX},+\operatorname{mar}(\mathrm{NS})$ & $M A[10 / 20]$ & $\mathrm{N}[0 / 10]$ & $46, X X / 47, X X,+$ mar \\
\hline PPP (Cl) & $46, \mathrm{XY} / 47, \mathrm{XY},+\operatorname{mar}(\mathrm{NS})$ & $\mathrm{N}[0 / 20]$ & NMA [10/10] & \\
\hline AMA & $46, \mathrm{XY} / 47, \mathrm{XY},+$ mar $(\mathrm{BS})$ & $\mathrm{MA}[12 / 18]$ & $\mathrm{MA}[9 / 12]$ & $46, X Y / 47, X Y,+m a r$ \\
\hline AMA & $46, X X / 47, X X,+\operatorname{mar}(\mathrm{MS})$ & MA [5/16] & $N[0 / 15]$ & \\
\hline AMA & $46, X X / 47, X X,+\operatorname{mar}(N S)$ & MA [5/19] & $N[0 / 14]$ & \\
\hline PBS & $46, X Y / 47, X Y,+i(18 p)$ & NMA [16/16] & $N[0 / 14]$ & \\
\hline AMA & $46, X X / 47, X X,+i(13 q)$ & $\mathrm{MA}[4 / 20]$ & $\mathrm{N}[0 / 12]$ & \\
\hline AMA & $46, X Y / 47, X Y,+i(13 q)$ & $\mathrm{MA}[4 / 36]$ & $N[0 / 7]$ & \\
\hline $\begin{array}{l}\text { MA } \\
\text { AMA }\end{array}$ & $\begin{array}{l}46, X X / 46, X X, i(21 q) \\
46, X X / 46, X X, i(13)(q 10)\end{array}$ & $\begin{array}{l}\mathrm{N}[0 / 18] \\
\mathrm{MA}[12 / 17]\end{array}$ & $\begin{array}{l}\text { MA [2/14] } \\
N[0 / 18]\end{array}$ & \\
\hline PPP (DW) & $46, X Y / 46, X Y, r(4)$ & NMA [21/21] & $\mathrm{N}[0 / 10]$ & \\
\hline & $46, X Y / 46, X Y, r(9)$ & $\mathrm{N}[0 / 25]$ & NMA [7/7] & \\
\hline PNT & $46, X Y / 46, X Y, \operatorname{del}(4 p)$ & NMA [18/18] & $\mathrm{N}[0 / 12]$ & \\
\hline AMA & $46, X X / 46, X X, \operatorname{del}(4 p)$ & MA [21/68] & $N[0 / 6]$ & \\
\hline AMA & $46 X Y / 46, X Y, \operatorname{del}(4)(q 32-q t e r)$ & $\mathrm{N}[0 / 25]$ & MÁ [4/6] & \\
\hline AMA & $46, X X / 46, X X, \operatorname{del}(11 q)$ & $N[0 / 24]$ & NMA [6/6 ] & \\
\hline AMA & $46, X X / 46, X X, \operatorname{del}(9 q)$ & $\mathrm{MA}[6 / 21]$ & $\mathrm{N}[0 / 20]$ & \\
\hline & $46 X Y / 46, X Y, \operatorname{del}(14 q)$ & $N[0 / 24]$ & MÄ [2/7] & \\
\hline AMA & $46 \mathrm{XX} / 46, \mathrm{XX}$, del(8)(q21.1-qter) & $N[0 / 18]$ & MA [3/13] & \\
\hline AMA & $46, X X / 46, X X, \operatorname{dup}(12)$ & $N[0 / 21]$ & MA [3/9] & \\
\hline AMA & $46, X Y / 46, X Y, \operatorname{add}(9 q)$ & MA [16/27] & $\mathrm{N}[0 / 11]$ & \\
\hline AMA & $46, X X / 46, X X$, add $(11 q)$ & MA [14/20] & $N[0 / 10]$ & \\
\hline AMA & $46, X Y / 46, X Y$, add $(16 p)$ & $\mathrm{N}[0 / 25]$ & NMA [7/7] & \\
\hline AMA & $46, X Y / 46, X Y, \operatorname{add}(2 q)$ & NMA [19/19] & $\mathrm{N}[0 / 12]$ & \\
\hline A & $46, X X / 46, X X$, add $(8 p)$ & NMA [16/16] & $\mathrm{N}[0 / 14]$ & \\
\hline AMA & $46, X X / 46, X X$, add $(15 q)$ & $\mathrm{MA}[8 / 18]$ & $N[0 / 12]$ & \\
\hline AMA & $46, X X / 46, X X, t(12 ; 15)$ & $\mathrm{N}[0 / 21]$ & MA [4/9] & \\
\hline AMA & $46, \mathrm{XY} / 46, \mathrm{XY}, \mathrm{t}(9 ; 13)$ & $N[0 / 20]$ & MA [6/19] & \\
\hline AMA & $46, \mathrm{XY} / 46, \mathrm{XY}, \mathrm{t}(3 ; 6)$ & $N[0 / 24]$ & $\mathrm{MA}[2 / 6]$ & \\
\hline AMA & $46, X X / 46, X X, t(2 ; 22)$ & $\mathrm{N}[0 / 24]$ & $\mathrm{MA}[3 / 7]$ & \\
\hline AMA & $46, X X / 46, X X, t(1 q ; 16 q)$ & $N[0 / 22]$ & MA [5/8] & \\
\hline AMA & $46, \mathrm{XY} / 46, \mathrm{XY}, \mathrm{t}(10 ; 12)(\mathrm{q} 23.3 ; \mathrm{p} 13.3)$ & $\mathrm{N}[0 / 22]$ & NMÄ [8/8] & $46, X Y, t(10 ; 12)(q 23.3 ; p 13.3)$ \\
\hline AMA & $46, X Y / 46, X Y, t(4 q ; 11 q)$ & $N[0 / 24]$ & NMA [6/6] & \\
\hline AMA & $46, \mathrm{XX} / 46, \mathrm{XX}, \mathrm{t}(9 ; 21)$ & $\mathrm{N}[0 / 18]$ & $\mathrm{MA}[4 / 12]$ & \\
\hline AMA & $46, \mathrm{XX} / 46, \mathrm{XX}, \mathrm{t}(3 ; 11)$ & $N[0 / 19]$ & $\mathrm{MA}[4 / 17]$ & \\
\hline AMA & $46, \mathrm{XX} / 46, \mathrm{XX}, \mathrm{t}(1 ; 14)$ & $\mathrm{MA}[8 / 34]$ & $N[0 / 6]$ & \\
\hline A & $46, X X / 45, X X, \operatorname{der}(13 ; 13)(q 10 ; q 10)$ & $\mathrm{MA}[4 / 23]$ & $N[0 / 18]$ & \\
\hline AMA & $46, X Y / 45, X Y, \operatorname{der}(14 ; 21)(q 10 ; q 10)$ & NMA $[27 / 27]$ & $N[0 / 12]$ & \\
\hline$P$ & 46XY/46,XY,rearr(6) & $N[0 / 24]$ & $\mathrm{MA}[4 / 6]$ & \\
\hline$A$ & $46, X Y / 46, X Y, \operatorname{rearr}(2)$ & $N[0 / 21]$ & $\mathrm{MA}[5 / 9]$ & \\
\hline
\end{tabular}

AMA, advanced maternal age; PNT, positive nuchal translucency; A, anxiety; PBS, positive biochemical screening; PPP, previous pathologic pregnancy; DW, dandy-walker; $\mathrm{Cl}$, cystic igroma; N, normal; MA, mosaic abnormality; NMA, nonmosaic abnormality; NS/MS/BS, non-/mono-/bi-satellited.

in amniocytes. The AF samples showing NMA (10 cases) were never associated with an MA in the cytotrophoblast and/or mesenchyme; all ten cases showed the homogeneous involvement of at least one placental tissue, preferentially the mesenchyme (nine cases). The most frequent abnormalities in amniocytes were sex chromosome aneuploidies (14/36 cases; 38.9\%). We found that trisomy 21 and 18 mosaicisms led to a similar risk of TFM, and no cases of CV trisomy 13 were confirmed in AF. These results are quite different from those published by Hahnemann and Vejerslev, ${ }^{23}$ who reported that TFM due to trisomy 21 mosaicism was the 


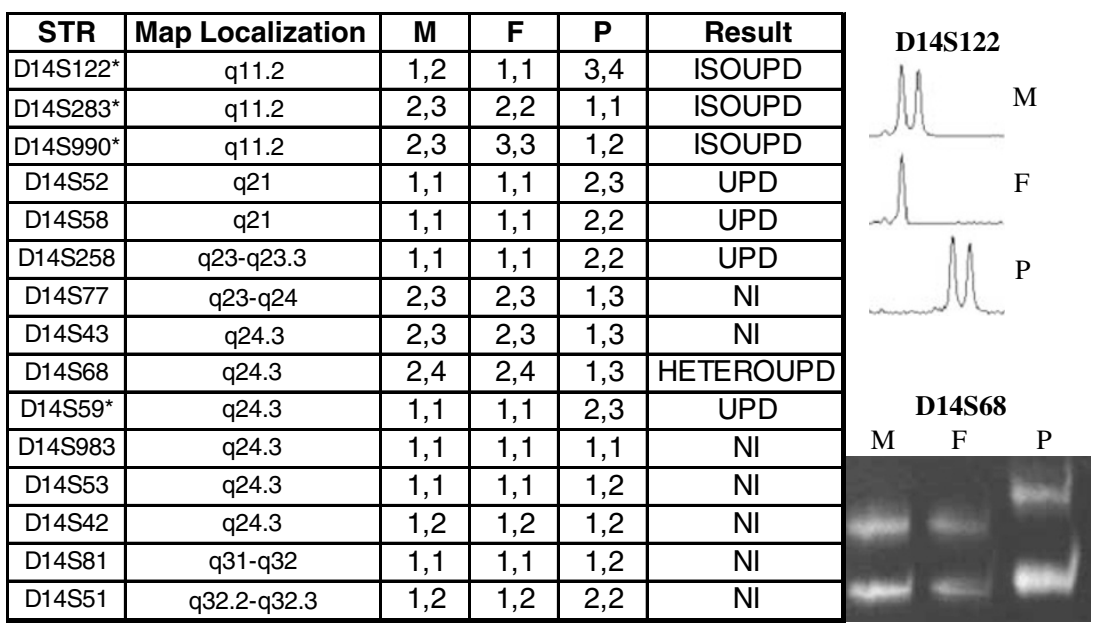

Figure 1 Molecular results of matUPD14 case. For each informative marker, maternal (M), fetal (F) and paternal (P) alleles are indicated. The fetus inherited alleles only from the mother and failed to inherit a paternal allele, consistent with a maternal disomy $14 .{ }^{*}$ Indicate STRs analysed by fluorescent capillary system.

most frequent, and confirmed fetal trisomy 13 in two of 15 cases.

When a type III CV mosaic involved trisomy 21, X/Y aneuploidies and supernumerary marker chromosomes, the abnormal cell line was always also present in amniocytes. Mosaic and nonmosaic sex chromosome aneuploidies strictly confined to the cytotrophoblast were never confirmed in AF. When nonmosaic trisomy 18 and $\mathrm{X} / \mathrm{Y}$ aneuploidies were present in the mesenchyme they were also always present in the fetus. ${ }^{25}$

Structural chromosome abnormalities Structural rearrangements with a $47,+$ mar karyotype carry a $27.3 \%$ risk of fetal confirmation, higher than the $3.2 \%$ risk in the case of a 46,rearr karyotype. In our study, the structural rearrangements other than supernumerary markers included only one case of type II CV mosaicism with a $46, X Y, t(10 ; 12)(q 23.3 ; p 13.3)$ cell line extended to the fetus. The literature of cases in which the cytotrophoblast, mesenchyme and amniocytes were analysed includes 14 cases of 46 ,rearr and 17 cases of $47,+$ mar. $^{3-8,26}$ In agreement with our findings, two of the TFM cases in the former group also showed the abnormal cell line in the mesenchyme, and five cases of TFM were recognised in the latter group.

We found four nonmosaic type II abnormalities involving structural rearrangements (46,rearr). In three of them, it was possible to analyse only six or seven metaphases, all showing an unbalanced karyotype; r(9), del(11q) and add(16p). In spite of the small number of metaphases we scored after culture, this finding could suggest the use of caution before any decision of pregnancy termination, for laboratories performing CV chromosome analysis using only long-term cultures.

\section{Confined placental mosaicisms and UPD risk assessment}

The distribution of CPMs involving trisomies is in line with that reported in other studies. ${ }^{23,27,28}$ Unlike Hahnemann and Vejerslev, ${ }^{23}$ we found one case of nonmosaic trisomy 22 in both placental tissues associated with a normal male karyotype in amniocytes. The CPMs due to structural rearrangements were equally distributed between types I and II.

A mosaic condition with a trisomic cell line is usually due to a nondisjunction error during meiosis I or II, followed by the postzygotic loss of the extra chromosome copy, which could theoretically lead to a diploid cell line with UPD in $33.3 \%$ of cases. We found an incidence of this condition of $1.96 \%(1 / 51)$, which is much lower than expected. One possible explanation is that the trisomic cell line may be mainly due to a mitotic mechanism, which is associated with a low risk of UPD and the formation of type I or II CPMs with a small percentage of abnormal cells. Type III and type I (with a high proportion of abnormal cells) often reflect a trisomic rescue of a meiotic error. ${ }^{13,28}$ In line with this hypothesis, type I and II CPMs were more frequent than type III in our cohort (163 vs 13 cases), thus explaining the low percentage of UPD. Our UPD14 case was a type I CPM with about 50\% of trisomic cells, possibly due to a trisomic rescue occurring in an early stage of embryonic development. Nevertheless, only eight metaphases were available after long-term culture, and so a type III CPM cannot be excluded with certainty.

The majority of published matUPD14 cases have been associated with a Robertsonian balanced translocation, and only eight have been described as originating from a trisomic zygote rescue. ${ }^{29-36}$ We here describe the third case of matUPD14 discovered after first trimester CV sampling 
and due to the correction of an initial trisomic zygote. ${ }^{30,32}$ The maternal isodisomy found at the pericentromeric STRs indicates the occurrence of a nondisjunction event during maternal M-II, and the heterodisomy for a telomeric locus may have been due to the occurrence of a meiotic crossover. The only finding observed in 15 th $+4 \mathrm{wg}$ was a decreased femoral length of $2 \mathrm{SD}$. As in the two previous reports, the indication for prenatal diagnosis was an advanced maternal age and the clinical condition of the fetus was very mild, thus indicating that a matUPD14 phenotype may not be easily recognised prenatally.

\section{References}

1 Vejerslev LO, Mikkelsen M: The European collaborative study on mosaicism in chorionic villus sampling: data from 1986 to 1987. Prenat Diagn 1989; 9: 575-588.

2 Medical Research Council working party on the evaluation of chorionic villus sampling: Medical Research Council European trial of chorionic villus sampling. Lancet 1991; 337: 1491-1499.

3 Teshima IE, Kalousek DK, Vekemans MJ et al: Canadian multicenter randomized clinical trial of chorion villus sampling and amniocentesis. chromosome mosaicism in CVS and amniocentesis samples. Prenat Diagn 1992; 12: 443-466.

4 Ledbetter DH, Zachary JM, Simpson JL et al: Cytogenetic results from the US Collaborative Study on CVS. Prenat Diagn 1992; 12: $317-345$.

5 Smidt-Jensen S, Lind AM, Permin M, Zachary JM, Lundsteen C, Philip J: Cytogenetic analysis of 2928 CVS samples and 1075 amniocenteses from randomized studies. Prenat Diagn 1993; 13: $723-740$

6 Wang BB, Rubin $\mathrm{CH}$, Williams 3rd J: Mosaicism in chorionic villus sampling: an analysis of incidence and chromosomes involved in 2612 consecutive cases. Prenat Diagn 1993; 13: 179-190.

7 Association of Clinical Cytogeneticists Working Party on Chorionic Villi in Prenatal Diagnosis: Cytogenetic analysis of chorionic villi for prenatal diagnosis: an ACC collaborative study of UK data. Prenatal Diagn 1994; 14: 363-379.

8 Wolstenholme J, Rooney DE, Davison EV: Confined placental mosaicism, IUGR, and adverse pregnancy outcome: a controlled retrospective UK collaborative survey. Prenat Diagn 1994; 14: $345-361$.

9 Stetten G, Escallon CS, South ST, McMichael JL, Saul DO, Blakemore KJ: Reevaluating confined placental mosaicism. Am J Med Genet 2004; 131: 232-239.

10 Simoni G, Fraccaro M: Does confined placental mosaicism affect the fetus? Hum Reprod 1992; 7: 139-140.

11 Kalousek DK, Barrett IJ, Gartner AB: Spontaneous abortion and confined chromosomal mosaicism. Hum Genet 1992; 88: $642-646$

12 Simoni G, Sirchia SM: Confined placental mosaicism. Prenat Diagn 1994; 14: 1185-1189.

13 Robinson WP, Barrett IJ, Bernard L et al: Meiotic origin of trisomy in confined placental mosaicism is correlated with presence of fetal uniparental disomy, high levels of trisomy in trophoblasts, and increased risk of fetal intrauterine growth restriction. $\mathrm{Am} \mathrm{J}$ Hum Genet 1997; 60: 917-927.

14 Engel E, DeLozier-Blanchet CD: Uniparental disomy, isodisomy, and imprinting: probable effects in man and strategies for their detection. Am J Med Genet 1991; 40: 432-439.

15 Kalousek DK: Pathogenesis of chromosomal mosaicism and its effect on early human development. Am J Med Genet 2000; 91: $39-45$.

16 Spence JE, Perciaccante RG, Greig GM et al: Uniparental disomy as a mechanism for human genetic disease. Am J Hum Genet 1988; 42: $217-226$.
17 Voss R, Ben-Simon E, Avital A et al: Isodisomy of chromosome 7 in a patient with cystic fibrosis: could uniparental disomy be common in humans? Am J Hum Genet 1989; 45: 373-380.

18 Kalousek DK, Langlois S, Barrett I et al: Uniparental disomy for chromosome 16 in humans. Am J Hum Genet 1993; 52: $8-16$.

19 Kalousek DK, Barrett I: Genomic imprinting related to prenatal diagnosis. Prenat Diagn 1994; 14: 1191-1201.

20 Ledbetter DH, Engel E: Uniparental disomy in humans: development of an imprinting map and its implications for prenatal diagnosis. Hum Mol Genet 1995; 4: Spec No 1757-1764.

21 Eggermann T, Zerres K, Eggermann K, Moore G, Wollmann HA: Uniparental disomy: clinical indications for testing in growth retardation. Eur J Pediatr 2002; 161: 305-312.

22 Grati FR, Sirchia SM, Garagiola I et al: Losses of heterozygosity in oral and oropharyngeal epithelial carcinomas. Cancer Genet Cytogenet 2000; 118: 57-61.

23 Hahnemann JM, Vejerslev LO: European collaborative research on mosaicism in CVS (EUCROMIC) - fetal and extrafetal cell lineages in 192 gestations with CVS mosaicism involving single autosomal trisomy. Am J Med Genet 1997; 70: 179-187.

24 Daniel A, Wu Z, Darmanian A et al: Issues arising from the prenatal diagnosis of some rare trisomy mosaics - the importance of cryptic fetal mosaicism. Prenat Diagn 2004; 24: 524-536.

25 Smith K, Lowther G, Maher E, Hourihan T, Wilkinson T, Wolstenholme J: The predictive value of findings of the common aneuploidies, trisomies 13,18 and 21 , and numerical sex chromosome abnormalities at CVS: experience from the ACC UK Collaborative Study. Association of Clinical Cytogeneticists Prenatal Diagnosis Working Party. Prenat Diagn 1999; 19: 817-826.

26 Miny P, Hammer P, Gerlach B et al: Mosaicism and accuracy of prenatal cytogenetic diagnoses after chorionic villus sampling and placental biopsies. Prenat Diagn 1991; 11: 581-589.

27 Wolstenholme J: An audit of trisomy 16 in man. Prenat Diagn 1995; 15: 109-121.

28 Wolstenholme J: Confined placental mosaicism for trisomies 2, 3 , $7,8,9,16$, and 22: their incidence, likely origins, and mechanisms for cell lineage compartmentalization. Prenat Diagn 1996; 16: 511-524.

29 Sirchia SM, De Andreis C, Pariani S et al: Chromosome 14 maternal uniparental disomy in the euploid cell line of a fetus with mosaic 46,XX/47,XX,+14 karyotype. Hum Genet 1994; 94: $355-358$.

30 Ralph A, Scott F, Tiernan C et al: Maternal uniparental isodisomy for chromosome 14 detected prenatally. Prenat Diagn 1999; 19: $681-684$.

31 Fokstuen S, Ginsburg C, Zachmann M, Schinzel A: Maternal uniparental disomy 14 as a cause of intrauterine growth retardation and early onset of puberty. I Pediatr 1999; 134: 689-695.

32 Sanlaville D, Aubry MC, Dumez Y et al: Maternal uniparental heterodisomy of chromosome 14: chromosomal mechanism and clinical follow up. J Med Genet 2000; 37: 525-528.

33 Towner DR, Shaffer LG, Yang SP, Walgenbach DD: Confined placental mosaicism for trisomy 14 and maternal uniparental disomy in association with elevated second trimester maternal serum human chorionic gonadotrophin and third trimester fetal growth restriction. Prenat Diagn 2001; 21: 395-398.

34 Aretz S, Raff R, Woelfle J, Zerres K, Esser M, Propping P et al: Maternal uniparental disomy 14 in a 15 -year-old boy with normal karyotype and no evidence of precocious puberty. Am J Med Genet A 2005; 135: 336-338.

35 Cox H, Bullman H, Temple IK: Maternal UPD(14) in the patient with a normal karyotype: clinical report and a systematic search for cases in samples sent for testing for Prader-Willi syndrome. Am J Med Genet A 2004; 127: 21-25.

36 Hordijk R, Wierenga H, Scheffer H, Leegte B, Hofstra RM, Stolte-Dijkstra I: Maternal uniparental disomy for chromosome 14 in a boy with a normal karyotype. J Med Genet 1999; 36: $782-785$. 